\title{
Algebraic Cryptanalysis of a Quantum Money Scheme: The Noise-Free Case
}

\author{
Marta Conde Pena ${ }^{1(凶)}$, Jean-Charles Faugère ${ }^{2,3,4}$, and Ludovic Perret ${ }^{2,3,4}$ \\ ${ }^{1}$ Institute of Physical and Information Technologies (ITEFI) - Spanish National \\ Research Council (CSIC), Madrid, Spain \\ marta.conde@iec.csic.es \\ 2 Sorbonne Universités, UPMC Univ Paris 06, POLSYS, UMR 7606, LIP6, 75005 \\ Paris, France \\ 3 INRIA, Paris-Rocquencourt Center, POLSYS Project, Paris, France \\ ${ }^{4}$ CNRS, UMR 7606, LIP6, 75005 Paris, France \\ jean-charles.faugere@inria.fr, ludovic.perret@lip6.fr
}

\begin{abstract}
We investigate the Hidden Subspace Problem $\left(\mathrm{HSP}_{q}\right)$ over $\mathbb{F}_{q}$ :

Input $: p_{1}, \ldots, p_{m}, q_{1}, \ldots, q_{m} \in \mathbb{F}_{q}\left[x_{1}, \ldots, x_{n}\right]$ of degree $d \geq 3$ (and $n \leq m \leq 2 n)$.
\end{abstract}

Find : a subspace $A \subset \mathbb{F}_{q}{ }^{n}$ of dimension $n / 2$ ( $n$ is even) such that

$$
p_{i}(A)=0 \forall i \in\{1, \ldots, m\} \text { and } q_{j}\left(A^{\perp}\right)=0 \forall j \in\{1, \ldots, m\},
$$

where $A^{\perp}$ denotes the orthogonal complement of $A$ with respect to the usual scalar product in $\mathbb{F}_{q}$.

This problem underlies the security of the first public-key quantum money scheme that is proved to be cryptographically secure under a non quantum but classic hardness assumption. This scheme was proposed by S. Aaronson and P. Christiano [1] at STOC'12. In particular, it depends upon the hardness of $\mathrm{HSP}_{2}$. More generally, Aaronson and Christiano left as an open problem to study the security of the scheme for a general field $\mathbb{F}_{q}$. We present a randomized polynomial-time algorithm that solves the $\mathrm{HSP}_{q}$ for $q>d$ with success probability $\approx 1-1 / q$. So, the quantum money scheme extended to $\mathbb{F}_{q}$ is not secure for big $q$. Finally, based on experimental results and a structural property of the polynomials that we prove, we conjecture that there is also a randomized polynomialtime algorithm solving the $\mathrm{HSP}_{2}$ with high probability. To support our theoretical results we also present several experimental results confirming that our algorithms are very efficient in practice. We emphasize that [1] proposes a non-noisy and a noisy version of the public-key quantum money scheme. The noisy version of the quantum money scheme remains secure.

\section{Introduction}

The no-cloning theorem in quantum mechanics states the impossibility of creating identical copies of an unknown arbitrary quantum money state. In [20],

(C) International Association for Cryptologic Research 2015

J. Katz (Ed.): PKC 2015, LNCS 9020, pp. 194-213, 2015.

DOI: $10.1007 / 978-3-662-46447-2 \_9$ 
Wiesner suggested to take advantage of this physical law in order to construct a scheme for (quantum) money that could not be counterfeited. The initial work of Wiesner has been then followed by several papers that try to improve the initial idea of $[20]$, i.e. $[5,17,18]$. This line of research culminated with the proposal of Aaronson and Christiano [1] at STOC'12 who proposed a public-key quantum money scheme.

A public-key quantum money scheme is a scheme in which anyone with a quantum device can verify if a banknote is valid rather than only the bank that issued it (in contrast to [20]). A public-key quantum money scheme based on knot theory was introduced in [12]. However, its security is not well understood. The scheme proposed by Aaronson and Christiano in [1] is the first that is public-key and proved to be cryptographically secure under a classical (as in nonquantum) hardness assumption. The scheme is based on hiding two orthogonal subspaces by expressing each of them as the common zeros of a set of appropriate random multivariate non-linear polynomials. In particular, its security relies on the assumption that the following problem is hard:

\section{Hidden Subspaces Problem $\left(\mathrm{HSP}_{q}\right)$}

Input : polynomials $p_{1}, \ldots, p_{m}, q_{1}, \ldots, q_{m} \in \mathbb{F}_{q}\left[x_{1}, \ldots, x_{n}\right]$ of degree $d \geq 3$, $n \leq m \leq 2 n$.

Find : a subspace $A \subset \mathbb{F}_{q}{ }^{n}$ of dimension $n / 2$ ( $n$ is even) such that

$$
p_{i}(A)=0 \forall i \in\{1, \ldots, m\} \text { and } q_{j}\left(A^{\perp}\right)=0 \forall j \in\{1, \ldots, m\},
$$

where $A^{\perp}$ denotes the orthogonal complement of $A$ with respect to the standard scalar product in $\mathbb{F}_{q}$.

We emphasize that in [1] the authors propose a non-noisy and a noisy version of the public-key quantum money scheme. In this paper we only consider the noise-free version of the quantum money scheme.

In particular, the non-noisy version of the quantum money scheme relies on the $\mathrm{HSP}_{2}$ and Aaronson and Christiano conjecture that it cannot be solved in polynomial time. They also state as an open problem the study of the scheme extended to a general field $\mathbb{F}_{q}$, which brings up the question of the hardness of $\mathrm{HSP}_{q}$.

We analyze the hardness of the $\mathrm{HSP}_{q}$. The main idea is to model the problem as a set of algebraic equations. Expressing elements as the common zeros of a set of random multivariate non-linear polynomials is the core of algebraic attacks, e.g. $[11,15,16]$. However, in this case we can exploit that there are two sets of public polynomials whose sets of zeros are two subspaces orthogonal to each other.

Aside from this quantum money scheme, the $\mathrm{HSP}_{q}$ has also interest as a general computer algebra problem closely related to the isomorphism of polynomials [19]. Given $\mathbf{p}=T \circ \mathbf{p}^{\prime} \circ S$, where $\mathbf{p}=\left(p_{1}, \ldots, p_{m}\right), \mathbf{p}^{\prime}=\left(p^{\prime}{ }_{1}, \ldots, p_{m}^{\prime}\right) \in$ $\mathbb{F}_{q}\left[x_{1}, \ldots, x_{n}\right]^{m}$ and $T, S$ are affine invertible transformations, the Isomorphism of Polynomials (IP) problem consists on recovering $T$ and $S$. The $\mathrm{HSP}_{q}$ can be seen as a slight modification of the isomorphism of polynomials problem where $\mathbf{p}=\mathbf{0}, T$ is the identity transformation and $S$ is linear but not invertible. 


\subsection{Main Results}

Our results mostly rely on Gröbner bases and linear algebra techniques. This is because we are capable of identifying the solution of the $\mathrm{HSP}_{q}$ as the unique solution of an overdetermined system of multivariate equations in $N=n^{2} / 4$ unknowns (Section 3.1, Proposition 5). The properties of this system are different for $q=2$ and for $q>d$, so we study separately both cases.

Our first main result (Section 3) solves an open problem presented in [1], which is the study of the $\mathrm{HSP}_{q}$ for $q \neq 2$. From the algebraic equations describing $\mathrm{HSP}_{q}$ we observe that we can extract a set of linear equations (Lemma 3). Due to the shape of the linear equations, we can prove that sufficiently many linearly independent ones can be extracted. This gives:

Theorem 1 (Section 3.2). Let $N=n^{2} / 4$. There is a randomized polynomialtime algorithm solving $\mathrm{HSP}_{q}$, for $q>d$, with complexity $\mathcal{O}\left(N^{\omega}\right)=\mathcal{O}\left(n^{2 \omega}\right)$, where $2 \leq \omega \leq 3$ is the linear algebra constant, and success probability

$$
\frac{\gamma_{q}(n / 2) \gamma_{q}(m)}{\gamma_{q}(m-n / 2)},
$$

$\gamma_{q}(k)$ being the probability that a random $k \times k$ matrix with entries in $\mathbb{F}_{q}$ is invertible. For $n$ big enough, the success probability is $\approx 1-1 / q$.

In Section 3.3 we report experimental results demonstrating that $\mathrm{HSP}_{q}$, with $q>d$, can be solved very efficiently. For $n \leq 20$, the algorithm requires less than $0.1 \mathrm{~s}$. for various $q$. We have implemented the algorithm using the MAGMA software [6]. The code is provided with the submission so that the results can be reproduced or conducted for bigger values of $n$.

Our second result is concerned with the $\mathrm{HSP}_{2}$ (Section 4). In this case our system does not contain, except with a small probability, linear equations and so the approach needs to be different. Still, in the case of the $\mathrm{HSP}_{2}$ we have an algebraic system of equations which is very overdetermined.

Proposition 1 (Section 4). Let $\left(\mathbf{p}=\left(p_{1}, \ldots, p_{m}\right), \mathbf{q}=\left(q_{1}, \ldots, q_{m}\right)\right) \in$ $\mathbb{F}_{2}\left[x_{1}, \ldots, x_{n}\right]^{m} \times \mathbb{F}_{2}\left[x_{1}, \ldots, x_{n}\right]^{m}$ be degree-d multivariate polynomials. Let $A \subset$ $\mathbb{F}_{2}^{n}$ be a vector subspace of dimension $n / 2$. If $A$ is a solution of $\mathrm{HSP}_{2}$ on $(\mathbf{p}, \mathbf{q}) \in \mathbb{F}_{2}\left[x_{1}, \ldots, x_{n}\right]^{m} \times \mathbb{F}_{2}\left[x_{1}, \ldots, x_{n}\right]^{m}$, then we can construct an algebraic system of equations $\mathrm{Sys}_{\mathrm{HSP}_{2}}$ over $\mathbb{F}_{2}$ in $N=n^{2} / 4$ variables with at most $2 m\left[\left(\begin{array}{c}n / 2 \\ 1\end{array}\right)+\left(\begin{array}{c}n / 2 \\ 2\end{array}\right)+\ldots+\left(\begin{array}{c}n / 2 \\ d\end{array}\right)\right]$ equations such that a systematic basis of $A$ vanishes $\operatorname{Sys}_{\mathrm{HSP}_{2}}$ with probability $\gamma_{2}(n / 2)$, where $\gamma_{2}(n / 2)$ denotes the probability that a random $n / 2 \times n / 2$ matrix with entries in $\mathbb{F}_{2}$ is invertible. For $n$ big enough, this is $\approx 1 / 2$.

So, we can still hope that the computation of a Gröbner basis of the system will be efficient. In Section 4.1 we run experiments to confirm this intuition. It appears that $\mathrm{Sys}_{\mathrm{HSP}_{2}}$ is much easier to solve than a semi-regular system of the same size. The MAGMA code of this part is also provided with the submission. Typically, we can solve in practice $\mathrm{Sys}_{\mathrm{HSP}_{2}}$ for $n \leq 18$ and $d=3$ in less than 
3 hours (for smaller $n$, we can solve in few minutes). For $n=18$, we have to solve a system of degree- 3 equations with 81 variables. In practice we observed that the maximum degree reached during the computation of a Gröbner basis of $\mathrm{Sys}_{\mathrm{HSP}_{2}}$ is bounded above by a small constant. Based on this observation we conjecture then that:

Conjecture 1. The degree of regularity is bounded above by $d+1$.

If this conjecture is true the following result is obtained:

Theorem 2. Let $N=n^{2} / 4$. There is a randomized polynomial-time algorithm solving $\mathrm{HSP}_{2}$ with a complexity of $\mathcal{O}\left(N^{\omega(d+1)}\right)=\mathcal{O}\left(n^{2 \omega(d+1)}\right)$, where $2 \leq \omega \leq$ 3 is the linear algebra constant, and success probability $\gamma_{2}(n / 2)$, where $\gamma_{2}(k)$ denotes the probability that a random $k \times k$ matrix with entries in $\mathbb{F}_{2}$ is invertible. For $n$ big enough, the success probability of the algorithm is $\approx 1 / 2$.

To support our assumption we analyze in Section 5 the structure of $\mathrm{Sys}_{\mathrm{HSP}_{2}}$. We prove a structural property (due to the orthogonality of the hidden subspaces) that allows to obtain equations of degree lower than $d$ from the public polynomials $\mathrm{Sys}_{\mathrm{HSP}_{2}}$ by performing simple manipulations on the initial system. In particular:

Proposition 2. We can easily generate $\mathcal{O}\left(\mathrm{m}^{2}\right)$ equations of degree $d-1$ which are linear combinations of the equations from $\mathrm{Sys}_{\mathrm{HSP}_{2}}$.

This means that a Gröbner computation on $\mathrm{Sys}_{\mathrm{HSP}_{2}}$ will generate at the very first step many equations of lower degree. This is known as a fall of degree and it is typically a behaviour which is not occurring in a random (i.e. semi-regular) system of equations. So, it is a first step towards proving our conjecture.

\subsection{Organization of the Paper}

In Section 2 we introduce some notation that will be used throughout the paper, we recall the basics of Gröbner bases and we describe the non-noisy version of the quantum money scheme of [1]. The first part of Section 3 is concerned with the general modeling of the $\mathrm{HSP}_{q}$ as a system of multivariate non-linear equations, and the second part is dedicated to obtain the algorithm of the first Theorem. Sections 4 and 5 are dedicated to the $\mathrm{HSP}_{2}$. In Section 4 we explain precisely why the behaviour of $\mathrm{HSP}_{q}$ is different for $q=2$ and for $q>d$, we report experimental results and we derive our conjecture which, if true, results in the second Theorem. Section 5 is the most technical one, in which we explain that equations of degree $<d$ can be obtained due to the orthogonality of the hidden subspaces.

\section{Preliminaries}

We first recall some basics of Gröbner bases as the main tool to approach nonlinear systems. Then we describe precisely our target problem and its relation 
with the quantum money scheme proposed in [1]. Before that we fix some general notation: we denote by $\mathbb{F}_{q}$ the finite field with $q$ elements, we set $\mathbf{x}=\left(x_{1}, \ldots, x_{n}\right)$ and $\mathbb{F}_{q}[\mathbf{x}]=\mathbb{F}_{q}\left[x_{1}, \ldots, x_{n}\right]$ to be the polynomial ring over $\mathbb{F}_{q}$ in the unknowns $x_{1}, \ldots, x_{n}$. We denote by $\mathrm{M}\left(\mathbb{F}_{q}[\mathbf{x}]\right)$ the set of monomials in $\mathbb{F}_{q}[\mathbf{x}], \mathrm{M}\left(\mathbb{F}_{2}[\mathbf{x}]\right)$ refers to the set of square-free monomials in $\mathbb{F}_{2}[\mathbf{x}]$ and $\mathrm{M}_{s}\left(\mathbb{F}_{q}[\mathbf{x}]\right)$ refers to the set of monomials of degree $s$ in $\mathbb{F}_{q}[\mathbf{x}]$. As usual, $\mathcal{M}_{k, \ell}\left(\mathbb{F}_{q}\right)$ denotes the set of $k \times \ell$ matrices with entries in $\mathbb{F}_{q}, \mathcal{M}_{k}\left(\mathbb{F}_{q}\right)$ denotes the square matrices of order $k$ with entries in $\mathbb{F}_{q}$, and $\mathrm{GL}_{k}\left(\mathbb{F}_{q}\right)$ the set of invertible matrices in $\mathcal{M}_{k}\left(\mathbb{F}_{q}\right)$.

\subsection{Basics of Computer Algebra}

As systems of multivariate non-linear equations are the key component of this work we recall some aspects of Gröbner bases computations [8-10]. Given a polynomial ideal over $\mathbb{F}_{q}$, say $\mathcal{I}=\left\langle f_{1}, \ldots, f_{s}\right\rangle=\left\{\sum_{i=1}^{s} f_{i} h_{i} \mid h_{1}, \ldots, h_{s} \in\right.$ $\left.\mathbb{F}_{q}[\mathbf{x}]\right\}$, Gröbner bases provide a way to obtain the variety $V_{q}(\mathcal{I})=\left\{x \in \mathbb{F}_{q} \mid\right.$ $f_{i}(\mathbf{x})=0$, for all $\left.1 \leq i \leq s\right\}$ by transforming the initial generators of the ideal into new generators with better properties, in the sense that computing the variety becomes simpler (this "better" set of generators is precisely the Gröbner basis).

The classic method to compute Gröbner bases is Buchberger's algorithm [8-10], but more efficient methods, such as $\mathrm{F}_{4}$ [13] and $\mathrm{F}_{5}$ [14], have been proposed. $F_{5}$ is considered to be one of the most efficient algorithms up to date for computing Gröbner bases. It uses linear algebra techniques and suppresses useless computations carried out in Buchberger's algorithm.

For increasing values of $\tilde{d}$, the $F_{5}$ algorithm successively reduces to row echelon form matrices of the form

$$
A_{\tilde{d}}=\begin{aligned}
& m_{1} f_{i_{1}} \\
& m_{2} f_{i_{2}} \\
& \ldots
\end{aligned}\left(\begin{array}{lll}
\ldots & \ldots & \ldots \\
\ldots & \ldots & \ldots \\
\ldots & \ldots & \ldots
\end{array}\right)
$$

where the columns are indexed by the monomials ordered decreasingly with respect to $<$, and $m_{j}$ are monomials such that $\operatorname{deg}\left(m_{j} f_{i_{j}}\right) \leq \tilde{d}$. At some point, for some $d$, the reduced row echelon form of the matrix $A_{d}$ contains a Gröbner basis. This maximum degree $d$ reached during a $\mathrm{F}_{5}$ computation is called the degree of regularity and it is an important parameter when assessing the running time of a Gröbner basis computation.

Systems verifying certain hypotheses are called semi-regular [3,4], and they are interesting due to two reasons. Firstly, because if a system is chosen at random it turns out to be semi-regular with high probability, and secondly because the degree of regularity is known for this kind of systems. In fact, if $\left\{f_{1}, \ldots, f_{s}\right\} \subset \mathbb{F}_{q}[\mathbf{x}]$ is a semi-regular system, where each $f_{i}$ has degree $d_{i}$, its degree of regularity is given by the index of the first non-positive coefficient of the power series 


$$
\sum_{k \geq 0} c_{k} z^{k}=\frac{\prod_{i=1}^{s}\left(1-z^{d_{i}}\right)}{(1-z)^{n}} .
$$

The time complexity of computing a Gröbner basis [2] is roughly given by the time spent carrying out the row echelon reduction of $A_{d}$, which is $\mathcal{O}\left(\left(\# A_{d}\right)^{\omega}\right)$, where $2 \leq \omega \leq 3$ is the linear algebra constant. Since the size of the matrix $A_{d}$ can be roughly approximated by $\mathcal{O}\left(n^{d}\right)$, this gives an overall complexity of $\mathcal{O}\left(n^{\omega d}\right)$.

\subsection{Definition of the Problem}

From now on we will always assume that $n$ is even. Recall that we are focusing on analyzing the hardness of the following problem:

\section{Hidden Subspaces Problem $\left(\mathrm{HSP}_{q}\right)$}

Input : $p_{1}, \ldots, p_{m}, q_{1}, \ldots, q_{m} \in \mathbb{F}_{q}[\mathbf{x}]$ of degree $d \geq 3(n \leq m \leq 2 n)$.

Find : a subspace $A \subset \mathbb{F}_{q}{ }^{n}$ of dimension $n / 2$ such that

$$
p_{i}(A)=0 \forall i \in\{1, \ldots, m\} \text { and } q_{j}\left(A^{\perp}\right)=0 \forall j \in\{1, \ldots, m\},
$$

where $A^{\perp}$ denotes the orthogonal complement of $A$ with respect to the standard scalar product in $\mathbb{F}_{q}$.

As mentioned in the introduction, $\mathrm{HSP}_{q}$ arises in relation to the security of the non-noisy version of the quantum money scheme proposed in [1]. The private key of this scheme is a subspace $A \subset \mathbb{F}_{q}{ }^{n}$, and the polynomials $p_{1}, \ldots, p_{m} \in \mathbb{F}_{q}[\mathbf{x}]$ (vanishing on $A$ ) and $q_{1}, \ldots, q_{m} \in \mathbb{F}_{q}[\mathbf{x}]$ (vanishing on $A^{\perp}$ ) are the public key. To output money, the bank queries an oracle to obtain a basis of $A$ and using this description of the subspace it generates a quantum state $\$$ which is the banknote. The verifying process is based on the fact that it is easy to check whether a given element is a zero of a polynomial or not.

The recovery of $A$ compromises the security of the scheme, so it becomes crucial that the $\mathrm{HSP}_{q}$ cannot be easily solved. It is conjectured in [1] that, for big enough $d$, there is no polynomial-time algorithm that solves $\mathrm{HSP}_{2}$ with success probability $\Omega\left(2^{-n / 2}\right)$.

Before proceeding any further we need to detail how the keys are generated. This is specified in [1]. The generation of a uniformly random subspace is clear by just choosing a full rank matrix in $\mathcal{M}_{n / 2, n}\left(\mathbb{F}_{q}\right)$. The generation of a uniformly random polynomial vanishing on a given subspace can be done in $\mathcal{O}\left(n^{d}\right)$-time:

Lemma 1 ([1]). Denote by $I_{d, A}$ the set of polynomials of degree $d$ that vanish on $A$, by $e_{i} \in \mathbb{F}_{q}^{n}$ the vector that has a 1 in its $i$-th position and 0 elsewhere, and by $E$ the subspace generated by the vectors $e_{1}, \ldots, e_{n / 2}$. We have:

1. A polynomial is in $I_{d, E}$ if and only if each of its monomials is divisible by an element in the set $\left\{x_{n / 2+1}, \ldots, x_{n}\right\}$. 
2. If $L$ is an invertible linear transformation on $I_{d, A}$, the function $p(\boldsymbol{x}) \rightarrow$ $p(\boldsymbol{x} L)$ maps $I_{d, A}$ to $I_{d, A L^{-1}}$.

Applying this lemma, one can generate polynomials vanishing on the appropriate subspace in the following way:

Proposition 3. (Vanishing polynomial) The generation of a uniformly random polynomial of degree $d$ vanishing on a given subspace $A$ consists of the following two steps:

1. Generate a polynomial $p(\boldsymbol{x})$ of degree d vanishing on $E$ : by lemma 1(1), this is done including each monomial of degree $d$ or lower independently and with probability $1 / 2$ if it is divisible by an element in the set $\left\{x_{n / 2+1}, \ldots, x_{n}\right\}$.

2. Transform the polynomial $p(\boldsymbol{x})$ into one vanishing on $A$ : considering the matrix $L$ of change of basis (i.e., $E=A L)$, the polynomial $p(x L)$ vanishes on $A$ by lemma 1(2).

We have performed all our experiments using Proposition 3.

\section{The $\mathrm{HSP}_{q}$, for $q>d$}

We analyze the hardness of the $\mathrm{HSP}_{q}$ for $q>d$. This is an open problem in [1] that arises when studying the security of the quantum money scheme extended to a general field $\mathbb{F}_{q}$. We conclude that the quantum money scheme extended to $\mathbb{F}_{q}$ is not secure for big $q$. First we show that, with a certain probability, the $\mathrm{HSP}_{q}$ can be modeled by a suitable set of non-linear equations. Then we prove that, with very high probability, enough linear equations that are linearly independent can be extracted from it. This results in a randomized polynomialtime algorithm for the $\mathrm{HSP}_{q}(q>d)$.

\subsection{General Modeling of $\mathrm{HSP}_{q}$}

In this part we show that the $\mathrm{HSP}_{q}$ can be rather naturally modeled as a set of algebraic equations. The first straightforward modeling presented is however not optimal as it includes many equivalent solutions. We show how we can use the structure of our problem to remove the unnecessary solutions.

We abuse notation and denote by $A$ either a subspace of $\mathbb{F}_{q}^{n}$ of dimension $n / 2$ or a matrix in $\mathcal{M}_{n / 2, n}\left(\mathbb{F}_{q}\right)$ whose rows are the elements of a basis of the subspace $A$.

Proposition 4. Let $\left(\mathbf{p}=\left(p_{1}, \ldots, p_{m}\right), \mathbf{q}=\left(q_{1}, \ldots, q_{m}\right)\right) \in \mathbb{F}_{q}[\boldsymbol{x}]^{m} \times \mathbb{F}_{q}[\boldsymbol{x}]^{m}$ be a degree-d instance of $\mathrm{HSP}_{q}$. Let $\left(y_{1}, \ldots, y_{n / 2}\right)$ be variables and $G=$ $\left(g_{i, j}\right)_{1 \leq i \leq n / 2,1 \leq j \leq n}, G^{\perp}=\left(g_{i, j}^{\perp}\right)_{1 \leq i \leq n / 2,1 \leq j \leq n}$ be formal matrices of size $n / 2 \times n$. $W e$ consider the system:

$$
\text { SysNaive }_{\mathrm{HSP}_{q}}=\left\{\operatorname{Coeff}\left(p_{i}, t\right), \operatorname{Coeff}\left(q_{j}, t\right) \mid \forall i, j \in\{1, \ldots, m\}, \forall t \in M\left(\mathbb{F}_{q}\left[y_{1}, \ldots, y_{n / 2}\right]\right)\right\}
$$


where $\operatorname{Coeff}\left(p_{i}, t\right)$ denotes the coefficient of $t \in \mathrm{M}\left(\mathbb{F}_{q}\left[g_{1,1}, \ldots, g_{n / 2, n}\right]\right)$ in $p_{i}\left(\left(y_{1}, \ldots, y_{n / 2}\right) \cdot G\right)$ and $\operatorname{Coeff}\left(q_{j}, t\right)$ the coefficient of $t \in \mathrm{M}\left(\mathbb{F}_{q}\left[g_{1,1}^{\perp}, \ldots, g_{n / 2, n}^{\perp}\right]\right)$ in $q_{j}\left(\left(y_{1}, \ldots, y_{n / 2}\right) \cdot G^{\perp}\right)$. SysNaive $\mathrm{HSP}_{q}$ is a system of $\mathcal{O}\left(n^{d+1}\right)$ algebraic equations over $\mathbb{F}_{q}$ in $n^{2}$ variables (the entries of $G$ and $G^{\perp}$ ).

Let $A \subset \mathbb{F}_{q}^{n}$ be a vector subspace of dimension $n / 2$. If $A$ is a solution of $\mathrm{HSP}_{q}$ on $(\mathbf{p}, \mathbf{q}) \in \mathbb{F}_{q}[\boldsymbol{x}]^{m} \times \mathbb{F}_{q}[\boldsymbol{x}]^{m}$ then the components of $A$ and $A^{\perp}$ vanish all the equations SysNaive $_{\mathrm{HSP}_{q}}$.

Proof. This is an immediate consequence of the fact that every element of the subspace $A$ (resp. $\left.A^{\perp}\right)$ can be expressed as $\left(y_{1}, \ldots, y_{n / 2}\right) A$ (resp. $\left(y_{1}, \ldots, y_{n / 2}\right) A^{\perp}$ ) . As a consequence, all the coefficients of the polynomials $p_{i}\left(\left(y_{1}, \ldots, y_{n / 2}\right) \cdot A\right)$ (resp. $p_{i}\left(\left(y_{1}, \ldots, y_{n / 2}\right) \cdot A^{\perp}\right)$ must be equal to zero.

It is easy to see that SysNaive $_{\mathrm{HSP}_{q}}$ has many solutions which are equivalent. If a vector subspace $A \subset \mathbb{F}_{q}^{n}$ is a solution of $\operatorname{HSP}_{q}$, then any basis of $A$ will be a solution SysNaive $\mathrm{HSP}_{q}$. It is then natural to define a canonical form of the solutions of $\mathrm{HSP}_{q}$.

Lemma 2. Let $\left(\mathbf{p}=\left(p_{1}, \ldots, p_{m}\right), \mathbf{q}=\left(q_{1}, \ldots, q_{m}\right)\right) \in \mathbb{F}_{q}[\boldsymbol{x}]^{m} \times \mathbb{F}_{q}[\boldsymbol{x}]^{m}$ be a degree-d instance of $\mathrm{HSP}_{q}$. Let $A \subset \mathbb{F}_{q}^{n}$ be a vector subspace of dimension $n / 2$. If $A$ is a solution of $\operatorname{HSP}_{q}$ on $(\mathbf{p}, \mathbf{q}) \in \mathbb{F}_{q}[\boldsymbol{x}]^{m} \times \mathbb{F}_{q}[\boldsymbol{x}]^{m}$, then for any $S \in$ $\mathrm{GL}_{n / 2}\left(\mathbb{F}_{q}\right), S \cdot A$ is a solution of $\mathrm{HSP}_{q}$ on $(\mathbf{p}, \mathbf{q}) \in \mathbb{F}_{q}[\boldsymbol{x}]^{m} \times \mathbb{F}_{q}[\boldsymbol{x}]^{m}$.

Proof. For all $i, 1 \leq i \leq m, p_{i}\left(\left(y_{1}, \ldots, y_{n / 2}\right) S \cdot A\right)=0$ holds as a consequence of $S$ being invertible. Also, since $(S A)^{\perp}=A^{\perp}$ (as $A^{\perp}(S A)^{T}=A^{\perp} A^{T} S^{T}=0$ considering that $\left.A^{\perp} A^{T}=0\right)$, it also holds that $q_{j}\left(\left(y_{1}, \ldots, y_{n / 2}\right)(S A)^{\perp}\right)=0$ for all $j, 1 \leq j \leq m$.

A direct consequence of Lemma 2 is that we can assume, with high probability, that a vector space solution $A$ of $\mathrm{HSP}_{q}$ is given in systematic form. This is, we can suppose that $A=(I \mid G)$, where $G \in \mathrm{GL}_{n / 2}\left(\mathbb{F}_{q}\right)$ and $I$ is the $n / 2 \times n / 2$ identity matrix. If $A$ has such a form then $A^{\perp}=\left(-G^{T} \mid I\right)$.

Fact 1. We recall that the probability that a random matrix in $\mathcal{M}_{n}\left(\mathbb{F}_{q}\right)$ is invertible is given by:

$$
\gamma_{q}(n)=\prod_{i=1}^{n}\left(1-\frac{1}{q^{i}}\right)
$$

It is well known that:

$$
\lim _{n \rightarrow \infty} \gamma_{q}(n)=1-\frac{1}{q}+\mathcal{O}\left(\frac{1}{q^{2}}\right) .
$$

For big values of $q, \gamma_{q}(n / 2)$ is close to 1 , which justifies the restriction on the shape of the subspace $A$.

We can now improve the modeling thanks to a canonical form of the solutions. We remove all the solutions of SysNaive $\mathrm{HSP}_{q}$ which correspond to equivalent bases. To do so, we generate a similar system of equations but with a smaller number of variables. 
Proposition 5. Let $\left(\mathbf{p}=\left(p_{1}, \ldots, p_{m}\right), \mathbf{q}=\left(q_{1}, \ldots, q_{m}\right)\right) \in \mathbb{F}_{q}[\boldsymbol{x}]^{m} \times \mathbb{F}_{q}[\boldsymbol{x}]^{m}$ be a degree-d instance of $\mathrm{HSP}_{q}$. Let $\left(y_{1}, \ldots, y_{n / 2}\right)$ be variables and $G=$ $\left(g_{i, j}\right)_{1 \leq i \leq n / 2,1 \leq j \leq n / 2}$ be a formal matrix of size $n / 2 \times n / 2$ and $N=n^{2} / 4$. We consider the system:

$$
\operatorname{Sys}_{\mathrm{HSP}_{q}}=\left\{\operatorname{Coeff}\left(p_{i}, t\right), \operatorname{Coeff}\left(q_{j}, t\right) \mid \forall i, j \in\{1, \ldots, m\}, \forall t \in M\left(\mathbb{F}_{q}\left[y_{1}, \ldots, y_{n / 2}\right]\right)\right\}
$$

where Coeff $\left(p_{i}, t\right)$ denotes the coefficient of $t \in \mathrm{M}\left(\mathbb{F}_{q}\left[g_{1,1}, \ldots, g_{n / 2, n / 2}\right]\right)$ in $p_{i}\left(\left(y_{1}, \ldots, y_{n / 2}\right) \cdot(I \mid G)\right)$, and $\operatorname{Coeff}\left(q_{j}, t\right)$ the coefficient of $t \in$ $\mathrm{M}\left(\mathbb{F}_{q}\left[g_{1,1}, \ldots, g_{n / 2, n / 2}\right]\right)$ in $q_{j}\left(\left(y_{1}, \ldots, y_{n / 2}\right) \cdot\left(-G^{T} \mid I\right)\right) . \operatorname{Sys}_{\mathrm{HSP}_{q}}$ is a system of $\mathcal{O}\left(n^{d+1}\right)$ algebraic equations over $\mathbb{F}_{q}$ in $N$ variables (the entries of $G$ ).

Let $A \subset \mathbb{F}_{q}^{n}$ be a vector subspace of dimension $n / 2$. If $A$ is a solution of $\mathrm{HSP}_{q}$ on $(\mathbf{p}, \mathbf{q}) \in \mathbb{F}_{q}[\boldsymbol{x}]^{m} \times \mathbb{F}_{q}[\boldsymbol{x}]^{m}$, then $A$ admits with probability $\gamma_{q}(n / 2)$ a basis in systematic form whose components vanish all the equations $\mathrm{Sys}_{\mathrm{HSP}_{q}}$.

Proof. This follows easily from Lemma 2 and Proposition 4.

So, Lemma 2 permitted to divide by 4 the number of variables that we have to consider.

\subsection{Randomized Polynomial-Time Algorithm for $\mathrm{HSP}_{q}$, with $q>d$}

According to Proposition 5, solving $\mathrm{HSP}_{q}$ is equivalent with high probability (w.h.p) to solve the non-linear system $\operatorname{Sys}_{\mathrm{HSP}_{q}}$. In this part we show that the non-linear system can be solved in polynomial-time. This is due to the fact that we can extract from $\mathrm{Sys}_{\mathrm{HSP}_{q}}$ sufficiently many linear equations that are linearly independent. These sufficiently many equations can be obtained with high probability considering only the polynomials $p_{1}, \ldots, p_{m}$ vanishing on $A$.

Lemma 3. Let $\left(\mathbf{p}=\left(p_{1}, \ldots, p_{m}\right), \mathbf{q}=\left(q_{1}, \ldots, q_{m}\right)\right) \in \mathbb{F}_{q}[\boldsymbol{x}]^{m} \times \mathbb{F}_{q}[\boldsymbol{x}]^{m}$ be $a$ degree-d instance of $\operatorname{HSP}_{q}$. Let $p_{i}^{(1)}$ (resp. $\left.q_{i}^{(1)}\right)$ be the homogeneous component of degree 1 of $p_{i}$ (resp. $\left.q_{i}\right)$, that is:

$$
\begin{aligned}
& p_{i}{ }^{(1)}=\sum_{j=1}^{n} \lambda_{i, j}^{p} x_{j}, \quad \text { where } \lambda_{i, 1}, \ldots, \lambda_{i, n} \in \mathbb{F}_{q}, \\
& q_{i}^{(1)}=\sum_{j=1}^{n} \lambda_{i, j}^{q} x_{j}, \quad \text { where } \lambda_{i, 1}, \ldots, \lambda_{i, n} \in \mathbb{F}_{q} .
\end{aligned}
$$

For $i \in\{1, \ldots, m\}$ and $k \in\{1, \ldots, n / 2\}$, the linear equations:

$$
\left\{\begin{array}{l}
\sum_{j=1}^{n / 2} \lambda_{i, j+n / 2}^{p} g_{k, j}+\lambda_{i, k}^{p} \\
\sum_{j=1}^{n / 2}-\lambda_{i, j}^{q} g_{j, k}+\lambda_{i, k+n / 2}^{q}
\end{array}\right.
$$

are in $\mathrm{Sys}_{\mathrm{HSP}}$. 
Proof. Let $G=\left(g_{i, j}\right)_{1 \leq i, j \leq n / 2}$ be a formal matrix. If we expand the products $\left(y_{1}, \ldots, y_{n / 2}\right)(I \mid G)$ and $\left(y_{1}, \ldots, y_{n / 2}\right)\left(-G^{T} \mid I\right)$, we can see that $\mathrm{Sys}_{\mathrm{HSP}_{q}}$ is obtained from the coefficients of:

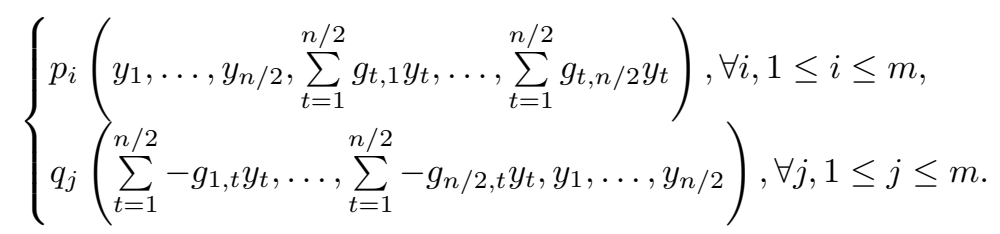

Since $q>d$ and so there are no reductions modulo the field equations, it is clear that the equations $\operatorname{Coeff}\left(p_{i}, y_{1}\right), \ldots, \operatorname{Coeff}\left(p_{i}, y_{n / 2}\right), \operatorname{Coeff}\left(q_{i}, y_{1}\right), \ldots, \operatorname{Coeff}\left(q_{i}, y_{n / 2}\right)$ are linear for all $i \in\{1, \ldots, m\}$. Taking into account the expressions of $p_{i}^{(1)}$ and $q_{i}^{(1)}$ as well as $(5)$, we have that for $k=1,2, \ldots, n / 2$ :

$$
\begin{aligned}
& \operatorname{Coeff}\left(p_{i}, y_{k}\right)=\lambda_{i, k}^{p}+\sum_{j=1}^{n / 2} \lambda_{i, j+n / 2}^{p} g_{k, j}, \\
& \operatorname{Coeff}\left(q_{i}, y_{k}\right)=\lambda_{i, k+n / 2}^{q}-\sum_{j=1}^{n / 2} \lambda_{i, j}^{q} g_{j, k},
\end{aligned}
$$

as required.

Let $N=n^{2} / 4$. Since $m \geq n$, the system of linear equations in Lemma 3 is already overdetermined with at most $2 m n / 2=m n \geq 4 N$ linear equations versus $N$ unknowns. We show now that among these (at most) $m n$ linear equations there are, with high probability, at least $N$ linearly independent ones, enough to solve it.

Lemma 4. Let $\left(\mathbf{p}=\left(p_{1}, \ldots, p_{m}\right), \mathbf{q}=\left(q_{1}, \ldots, q_{m}\right)\right) \in \mathbb{F}_{q}[\boldsymbol{x}]^{m} \times \mathbb{F}_{q}[\boldsymbol{x}]^{m}$ be a degree-d instance of $\mathrm{HSP}_{q}$. With probability $\frac{\gamma_{q}(m)}{\gamma_{q}(m-n / 2)}$, we can extract from $\mathrm{Sys}_{\mathrm{HSP}_{q}}$ at least $N=n^{2} / 4$ linear equations that are linearly independent.

Proof. The $m n \times N$ matrix of coefficients associated to the linear system specified in Lemma 3, whose columns are the unknowns $g_{1}, \ldots, g_{n / 2}, \ldots, g_{N-n / 2}, \ldots, g_{N}$, is the following:

$$
\left(\begin{array}{cccccccccc}
\ldots & \ldots & \ldots & \ldots & \ldots & \ldots & \ldots & \ldots & \ldots & \ldots \\
\lambda_{i, n / 2+1}^{p} & \ldots & \lambda_{i, n}^{p} & 0 & \ldots & 0 & \ldots & 0 & \ldots & 0 \\
0 & \ldots & 0 & \lambda_{i, n / 2+1}^{p} & \ldots & \lambda_{i, n}^{p} & \ldots & 0 & \ldots & 0 \\
\ldots & \ldots & \ldots & \ldots & \ldots & \ldots & \ldots & \ldots & \ldots & \ldots \\
0 & \ldots & 0 & 0 & \ldots & 0 & \ldots & \lambda_{i, n / 2+1}^{p} & \ldots & \lambda_{i, n}^{p} \\
\ldots & \ldots & \ldots & \ldots & \ldots & \ldots & \ldots & \ldots & \ldots & \ldots \\
-\lambda_{j, 1}^{q} & \ldots & 0 & -\lambda_{j, 2}^{q} & \ldots & 0 & \ldots & -\lambda_{j, n / 2}^{q} & \ldots & 0 \\
\ldots & \ldots & \ldots & \ldots & \ldots & \ldots & \ldots & \ldots & \ldots & \ldots \\
0 & \ldots & -\lambda_{j, 1}^{q} & 0 & \ldots & -\lambda_{j, 2}^{q} & \ldots & 0 & \ldots & -\lambda_{j, n / 2}^{q} \\
\ldots & \ldots & \ldots & \ldots & \ldots & \ldots & \ldots & \ldots & \ldots & \ldots
\end{array}\right)
$$


We restrict our attention to the following $m n / 2 \times N$ submatrix containing the equations $\operatorname{Coeff}\left(p_{i}, y_{j}\right)$ only, for $i \in\{1, \ldots, m\}$ and $j \in\{1, \ldots, n / 2\}$ :

$$
\left(\begin{array}{cccccccccc}
\ldots & \ldots & \ldots & \ldots & \ldots & \ldots & \ldots & \ldots & \ldots & \ldots \\
\lambda_{i, n / 2+1}^{p} & \ldots & \lambda_{i, n}^{p} & 0 & \ldots & 0 & \ldots & 0 & \ldots & 0 \\
0 & \ldots & 0 & \lambda_{i, n / 2+1}^{p} & \ldots & \lambda_{i, n}^{p} & \ldots & 0 & \ldots & 0 \\
\ldots & \ldots & \ldots & \ldots & \ldots & \ldots & \ldots & \ldots & \ldots & \ldots \\
0 & \ldots & 0 & 0 & \ldots & 0 & \ldots & \lambda_{i, n / 2+1}^{p} & \ldots & \lambda_{i, n}^{p} \\
\ldots & \ldots & \ldots & \ldots & \ldots & \ldots & \ldots & \ldots & \ldots & \ldots
\end{array}\right)
$$

We see that due to its particular shape it has rank $N$ if there exists an $n / 2 \times n / 2$ invertible submatrix in the following $m \times n / 2$ matrix:

$$
\left(\begin{array}{cccc}
\lambda_{1, n / 2+1}^{p} & \lambda_{1, n / 2+2}^{p} & \ldots & \lambda_{1, n}^{p} \\
\lambda_{2, n / 2+1}^{p} & \lambda_{2, n / 2+2}^{p} & \ldots & \lambda_{2, n}^{p} \\
\ldots & \cdots & \ldots & \ldots \\
\lambda_{m, n / 2+1}^{p} & \lambda_{m, n / 2+2}^{p} & \ldots & \lambda_{m, n}^{p}
\end{array}\right)
$$

this is, if the matrix (6) is of maximum rank. Since the coefficients of the matrix are uniformly random, the probability that an $m \times n / 2$ matrix has maximum rank is, according to [7], precisely

$$
\frac{\left(1-\frac{1}{q}\right) \ldots\left(1-\frac{1}{q^{m}}\right)}{\left(1-\frac{1}{q}\right) \ldots\left(1-\frac{1}{q^{m-n / 2}}\right)}=\frac{\gamma_{q}(m)}{\gamma_{q}(m-n / 2)} .
$$

Considering that the shape of $A$ is of the restricted form we assumed with probability $\gamma_{q}(n / 2)$ and that the system above can be solved successfully with probability $\frac{\gamma_{q}(m)}{\gamma_{q}(m-n / 2)}$, the following theorem sums up the results of this section:

Theorem 3. Let $q>d$. There is a randomized polynomial-time algorithm solving $\mathrm{HSP}_{q}$ in:

$$
\mathcal{O}\left(n^{2 \omega}\right)
$$

where $2 \leq \omega \leq 3$ is the linear algebra constant, and with success probability

$$
\frac{\gamma_{q}(n / 2) \gamma_{q}(m)}{\gamma_{q}(m-n / 2)}
$$

The success probability of our algorithm can be asymptotically approximated by 1 $1 / q$.

Proof. The algorithm to solve $\mathrm{HSP}_{q}$ is the following: 
Input: $p_{1}, \ldots, p_{m}, q_{1}, \ldots, q_{m} \in \mathbb{F}_{q}[\mathbf{x}]$ of degree $d \geq 3$.

Construct the first set of equations of the linear system of Lemma 3.

Solve it.

Return this solution.

Taking into account that $\gamma_{q}(n)=\prod_{i=1}^{n}\left(1-\frac{1}{q^{i}}\right)$ and that $\lim _{n \rightarrow \infty} \gamma_{q}(n)=1-\frac{1}{q}+$ $\mathcal{O}\left(\frac{1}{q^{2}}\right)$

$$
\lim _{n \rightarrow \infty} \frac{\gamma_{q}(n / 2) \gamma_{q}(m)}{\gamma_{q}(m-n / 2)}=1-\frac{1}{q}+\mathcal{O}\left(\frac{1}{q^{2}}\right)
$$

follows, and so the asymptotic success probability of our algorithm increases as we increase $q$.

\subsection{Experimental Results}

We report here our experimental results for $\mathrm{HSP}_{q}$, with $q>d$, obtained with the algorithm of Theorem 3. We have implemented the algorithm using the MAGMA software [6]. In the tables below, NextPrime $(k)$ is the Magma function that outputs the least prime number greater than $k$. Also, Time gen $_{\text {is }}$ the time needed to generate the instances, and Time is the time spent solving the linear system. Finally, $N=$ $n^{2} / 4$ is the number of unknowns in the linear system.

\begin{tabular}{|c|c|c|c|c|c|c|c|c|}
\hline \multicolumn{9}{|c|}{$d=3$} \\
\hline$n$ & & $q$ & $N$ & \multicolumn{2}{|c|}{ Time $_{\text {gen }}$} & Time & \multicolumn{2}{|c|}{ Memory } \\
\hline 10 & & 5 & 25 & \multicolumn{2}{|r|}{$1 \mathrm{~s}$} & $0.00 \mathrm{~s}$. & \multicolumn{2}{|c|}{$13 \mathrm{MB}$} \\
\hline 12 & & 5 & 36 & \multicolumn{2}{|r|}{$2 \mathrm{~s}$} & $0.00 \mathrm{~s}$. & \multicolumn{2}{|c|}{$12 \mathrm{MB}$} \\
\hline 20 & & 5 & 100 & \multicolumn{2}{|c|}{$135.1 \mathrm{~s}$} & $0.02 \mathrm{~s}$ & \multicolumn{2}{|c|}{$481 \mathrm{MB}$} \\
\hline 10 & \multicolumn{2}{|c|}{ NextPrime $\left(2^{16}\right)$} & 25 & \multicolumn{2}{|r|}{$1 \mathrm{~s}$} & $0.00 \mathrm{~s}$ & \multicolumn{2}{|c|}{$11 \mathrm{MB}$} \\
\hline 12 & \multicolumn{2}{|c|}{ NextPrime $\left(2^{16}\right)$} & 36 & \multicolumn{2}{|r|}{$4 \mathrm{~s}$} & $0.00 \mathrm{~s}$ & \multicolumn{2}{|c|}{$12 \mathrm{MB}$} \\
\hline \multirow[t]{8}{*}{20} & Nex & tPrime $\left(2^{16}\right)$ & 100 & & $44.7 \mathrm{~s}$ & 0.03 & & $\mathrm{MB}$ \\
\hline & \multicolumn{8}{|c|}{$d=4$} \\
\hline & $n$ & $q$ & & $N$ & \multicolumn{2}{|c|}{ Time $_{\text {gen }}$} & Time & Memory \\
\hline & 10 & 5 & & 25 & \multicolumn{2}{|c|}{$8 \mathrm{~s}$} & $0.00 \mathrm{~s}$ & $16 \mathrm{MB}$ \\
\hline & 12 & 5 & & 36 & \multicolumn{2}{|c|}{$40 \mathrm{~s}$} & $0.00 \mathrm{~s}$ & $16 \mathrm{MB}$ \\
\hline & 10 & NextPrime $(2$ & & 25 & \multicolumn{2}{|c|}{$18 \mathrm{~s}$} & $0.0 \mathrm{~s}$ & $22 \mathrm{MB}$ \\
\hline & 12 & NextPrime $(2$ & & 36 & \multicolumn{2}{|c|}{$107 \mathrm{~s}$} & $0.0 \mathrm{~s}$ & $22 \mathrm{MB}$ \\
\hline & 20 & NextPrime $(2$ & & 100 & \multicolumn{2}{|c|}{$5154.050 \mathrm{~s}$} & $0.02 \mathrm{~s}$ & $300 \mathrm{MB}$ \\
\hline
\end{tabular}

As expected from Theorem 3 the algorithm is very efficient. Note that even for small $q$, all experiments performed succeeded as the probability of obtaining sufficiently many linearly independent linear equations, $\gamma_{q}(m) / \lambda_{q}(m-n / 2)$, tends to 1 very quickly even for small values of $q$. Note that the running time of our algorithm 
is clearly dominated by the time spent in generating the instance. This is done in polynomial time ${ }^{1}$, so we can infer from the experiments that the algorithm runs in polynomial time, which is coherent with the theoretical results obtained.

\section{An Efficient Algorithm for Solving $\mathrm{HSP}_{2}$}

We consider in this part the special case of $\mathrm{HSP}_{2}$. As in Proposition 5, we can model $\mathrm{HSP}_{2}$ by a set of algebraic equations. However, the system for $q=2$ will have a different structure than $\mathrm{Sys}_{\mathrm{HSP}_{q}}$ due to reductions modulo the field equations that we now have to consider. In particular, it is no longer possible to extract linear equations. As a consequence, we have to adopt a different strategy for solving $\mathrm{HSP}_{2}$. First we adapt the modeling for $\mathrm{HSP}_{2}$ adding the field equations:

Proposition 6. Let $\left(\mathbf{p}=\left(p_{1}, \ldots, p_{m}\right), \mathbf{q}=\left(q_{1}, \ldots, q_{m}\right)\right) \in \mathbb{F}_{2}[\boldsymbol{x}]^{m} \times \mathbb{F}_{2}[\boldsymbol{x}]^{m}$ be a degree-d instance of $\mathrm{HSP}_{2}$. Let $\left(y_{1}, \ldots, y_{n / 2}\right)$ be variables and $G=\left(g_{i, j}\right)_{1 \leq i, j \leq n / 2}$ be a formal matrix and $N=n^{2} / 4$. We consider the system:

$$
\begin{array}{r}
\operatorname{Sys}_{\mathrm{HSP}_{2}}=\left\{\operatorname{Coeff}\left(p_{i}, t\right), \operatorname{Coeff}\left(q_{j}, t\right) \mid \forall i, j \in\{1, \ldots, m\}, \forall t \in M\left(\mathbb{F}_{2}\left[y_{1}, \ldots, y_{n / 2}\right]\right)\right\} \cup \\
\left\{g_{i, j}^{2}-g_{i, j} \mid 1 \leq i, j \leq n / 2\right\}
\end{array}
$$

where Coeff $\left(p_{i}, t\right)$ denotes the coefficient of $t \in \mathrm{M}\left(\mathbb{F}_{2}\left[g_{1,1}, \ldots, g_{n / 2, n / 2}\right]\right)$ in $p_{i}\left(\left(y_{1}, \ldots, y_{n / 2}\right) \cdot(I \mid G)\right)$, and $\operatorname{Coeff}\left(q_{j}, t\right)$ the coefficient of $t \in$ $\mathrm{M}\left(\mathbb{F}_{2}\left[g_{1,1}, \ldots, g_{n / 2, n / 2}\right]\right)$ in $q_{j}\left(\left(y_{1}, \ldots, y_{n / 2}\right) \cdot\left(G^{T} \mid I\right)\right)$. Sys $_{\mathrm{HSP}_{2}}$ is a system of at most $2 m\left[\left(\begin{array}{c}n / 2 \\ 1\end{array}\right)+\left(\begin{array}{c}n / 2 \\ 2\end{array}\right)+\ldots+\left(\begin{array}{c}n / 2 \\ d\end{array}\right)\right]$ algebraic equations over $\mathbb{F}_{2}$ in $N$ variables (the entries of $G$ ).

Let $A \subset \mathbb{F}_{2}^{n}$ be a vector subspace of dimension $n / 2$. If $A$ is a solution of $\mathrm{HSP}_{2}$ on $(\mathbf{p}, \mathbf{q}) \in \mathbb{F}_{2}[\boldsymbol{x}]^{m} \times \mathbb{F}_{2}[\boldsymbol{x}]^{m}$, then $A$ admits with probability $\gamma_{2}(n / 2)$ a basis in systematic form whose components vanish all the equations $\mathrm{Sys}_{\mathrm{HSP}_{2}}$.

Proof. Direct application of Proposition 5 and the fact that

$$
\#\left(\mathrm{M}\left(\mathbb{F}_{2}\left[y_{1}, \ldots, y_{n / 2}\right]\right)=\left(\begin{array}{c}
n / 2 \\
1
\end{array}\right)+\ldots+\left(\begin{array}{c}
n / 2 \\
d
\end{array}\right) .\right.
$$

Note that the equations of $\mathrm{Sys}_{\mathrm{HSP}_{2}}$ are of degree $d$ with high probability for big enough parameters. Indeed, let $G=\left(g_{i, j}\right)_{1 \leq i, j \leq n / 2}$ be a formal matrix of size $n / 2 \times n / 2$ and recall that $\mathrm{Sys}_{\mathrm{HSP}_{2}}$ is obtained from the coefficients of:

$$
\left\{\begin{array}{l}
p_{i}\left(y_{1}, \ldots, y_{n / 2}, \sum_{t=1}^{n / 2} g_{t, 1} y_{t}, \ldots, \sum_{t=1}^{n / 2} g_{t, n / 2} y_{t}\right), \forall i, 1 \leq i \leq m, \\
q_{j}\left(\sum_{t=1}^{n / 2}-g_{1, t} y_{t}, \ldots, \sum_{t=1}^{n / 2}-g_{n / 2, t} y_{t}, y_{1}, \ldots, y_{n / 2}\right), \forall j, 1 \leq j \leq m .
\end{array}\right.
$$

\footnotetext{
${ }^{1}$ Note that the generation of the instance is rather slow in practice, probably due to a non-optimal implementation of the Evaluate function in MAGMA for symbolic polynomials.
} 
Since we reduce modulo the field equations, the coefficient of a linear term is obtained from the linear terms of $p_{i}$ and $q_{j}$ but also from the coefficients of higher degree terms reduced modulo the field equations. So, it is expected with a high probability that $\mathrm{Sys}_{\mathrm{HSP}_{2}}$ has no linear equations.

However, although $\mathrm{Sys}_{\mathrm{HSP}_{2}}$ is non-linear it is greatly overdetermined. Thus we can expect that computing a Gröbner basis of $\mathrm{Sys}_{\mathrm{HSP}_{2}}$ can still be done efficiently.

\subsection{Experimental Results and Interpretation}

The goal of this part is to show that $\mathrm{Sys}_{\mathrm{HSP}_{2}}$ is indeed much easier to solve than a semi-regular system of the same size. Recall that if a system is semi-regular, its degree of regularity is given by the first non-positive coefficient of the power series specified in (2).

We report experiments run on a $2.93 \mathrm{GHz}$ Intel PC with $128 \mathrm{~Gb}$. of RAM with the MAGMA software [6] (V2.19-1) for the most disadvantageous choice of parameters (this is, $m=n$ ). We recall that MAGMA implements the $\mathrm{F}_{4}$ algorithm ([13]) for computing Gröbner bases.

The notation used in the table is the following: $n$ the number of variables of the public polynomials, $N=n^{2} / 4$ is the number of unknowns of the system in proposition $6, \mathrm{U}_{\text {eqs }}$ is the upper bound on the number of equations as specified in proposition $6, d_{\text {reg }}$ is the degree of regularity observed in practice, and $d_{\text {reg }}^{\text {sg }}$ is the theoretical degree of regularity treating the system as if it was semi-regular. The

\begin{tabular}{|c|c|c|c|c|c|c|}
\hline \multicolumn{7}{|c|}{$d=3$} \\
\hline$n$ & $N$ & $\mathrm{U}_{\text {eqs }}$ & $d_{\text {reg }}^{\text {sg }}$ & $d_{\text {reg }}$ & Time & Memory \\
\hline 8 & 16 & 224 & 4 & 3 & $1 \mathrm{~s}$ & $17 \mathrm{MB}$ \\
\hline 10 & 25 & 500 & 5 & 3 & $1 \mathrm{~s}$ & $20 \mathrm{MB}$ \\
\hline 12 & 36 & 984 & 5 & 3 & $2 \mathrm{~s}$ & $55 \mathrm{MB}$ \\
\hline 14 & 49 & 1764 & 5 & 4 & $136 \mathrm{~s}$ & $3 \mathrm{~Gb}$ \\
\hline 16 & 64 & 2944 & 6 & 4 & $2.30 \mathrm{~min}$ & $8 \mathrm{~GB}$ \\
\hline 18 & 81 & 4725 & 7 & 4 & $2 \mathrm{~h} 20$ & $80 \mathrm{~GB}$ \\
\hline
\end{tabular}

\begin{tabular}{|c|c|c|c|c|c|c|}
\hline \multicolumn{7}{|c|}{$d=4$} \\
\hline$n$ & $N$ & $\mathrm{U}_{\text {eqs }}$ & $d_{\text {reg }}^{\text {sg }}$ & $d_{\text {reg }}$ & Time & Memory \\
\hline 8 & 16 & 240 & 6 & 4 & $1 \mathrm{~s}$ & $20 \mathrm{MB}$ \\
\hline 10 & 25 & 600 & 6 & 4 & $1 \mathrm{~s}$ & $50 \mathrm{MB}$ \\
\hline 12 & 36 & 1344 & 7 & 5 & $38 \mathrm{~s}$ & $840 \mathrm{MB}$ \\
\hline 14 & 49 & 2744 & 8 & 5 & $66 \mathrm{~min}$ & $8 \mathrm{~GB}$ \\
\hline
\end{tabular}

first thing we observe is that the number of equations of our system coincides with the upper bound for the maximum number of possible equations. The experiments show that solving these systems is easier than if they were random: the degree of regularity observed in practice is indeed lower than the expected one, which suggests that there is an underlying structure that can be exploited. Furthermore, the degree of regularity stays bounded, so we conjecture the following:

Conjecture 1. The degree of regularity is bounded above by $d+1$.

If our conjecture is true, there is a randomized polynomial-time algorithm for $\mathrm{HSP}_{2}$ as follows: 
Theorem 4. Assuming Conjecture 1, there is a randomized polynomial-time algorithm (the computation of a Gröbner basis) solving degree-d instances of $\mathrm{HSP}_{2}$ with a complexity of

$$
\mathcal{O}\left(n^{2 \omega(d+1)}\right),
$$

where $2 \leq \omega \leq 3$ is the linear algebra constant, and success probability $\gamma_{2}(n / 2)$.

\section{$5 \quad$ Structural Low Degree Equations}

The goal of this part is to provide theoretical arguments supporting Conjecture 1. That is, we show that the system of algebraic equations of Proposition 6 has a very particular structure. We prove that suitable linear combinations of the equations will lead to equations of a lower degree. This is actually the first computation performed by a Gröbner basis algorithm on the system of Proposition 6. As a consequence, solving the system of degree $d$-equations from Proposition 6 reduces to solve a system with equations of degree $d$ and degree $d-1$. This is typically a behaviour which is not occurring in a random (i.e. semi-regular) system of equations and so it is a first step towards proving Conjecture 1.

Let $(p, q) \in \mathbb{F}_{2}[\mathbf{x}] \times \mathbb{F}_{2}[\mathbf{x}]$ be polynomials of degree $d$ such that $p$ vanishes on a vector subspace $A \subset \mathbb{F}_{2}^{n}$ of dimension $n / 2$ and $q$ vanishes on the orthogonal space $A^{\perp}$. Let $N_{d}=\left(\begin{array}{c}n / 2 \\ d\end{array}\right)$. We order lexicographically the monomials of degree $d$ in the rings

$$
\mathbb{F}_{2}\left[y_{1}, \ldots, y_{n / 2}\right], \mathbb{F}_{2}\left[x_{n / 2+1}, \ldots, x_{n}\right] \text {, and } \mathbb{F}_{2}\left[x_{1}, \ldots, x_{n / 2}\right] \text {. }
$$

We then denote by $t_{1}<\ldots<t_{N_{d}}, m_{1}<\ldots<m_{N_{d}}$ and $m^{\perp}{ }_{1}<\ldots<m^{\perp}{ }_{N_{d}}$ the respective monomials in ascending order. This way we can write

$$
\left\{\begin{array}{l}
p=\alpha_{1} m_{1}+\ldots+\alpha_{N_{d}} m_{N_{d}}+\tilde{p}, \alpha_{1}, \ldots, \alpha_{N_{d}} \in \mathbb{F}_{2}, \tilde{p} \in \mathbb{F}_{2}[\mathbf{x}] \backslash \mathrm{M}_{d}\left(\mathbb{F}_{2}\left[x_{\frac{n}{2}}+1, \ldots, x_{n}\right]\right), \\
q=\beta_{1} m^{\perp}{ }_{1}+\ldots+\beta_{N_{d}} m^{\perp} N_{d}+\tilde{q}, \beta_{1}, \ldots, \beta_{N_{d}} \in \mathbb{F}_{2}, \tilde{q} \in \mathbb{F}_{2}[\mathbf{x}] \backslash \mathrm{M}_{d}\left(\mathbb{F}_{2}\left[x_{1}, \ldots, x_{n / 2}\right]\right) .
\end{array}\right.
$$

Recall that the notation Coeff $(p, t)$ refers to the coefficient of $t \in \mathrm{M}\left(\mathbb{F}_{2}\left[g_{1,1}, \ldots\right.\right.$, $\left.\left.g_{n / 2, n / 2}\right]\right)$ occurring in $p\left(\left(y_{1}, \ldots, y_{n / 2}\right)(I \mid G)\right)$, and Coeff $(q, t)$ refers to the coefficient of $t \in \mathrm{M}\left(\mathbb{F}_{2}\left[g_{1,1}, \ldots, g_{n / 2, n / 2}\right]\right)$ occurring in $p\left(\left(y_{1}, \ldots, y_{n / 2}\right)\left(G^{T} \mid I\right)\right)$. We will denote by Coeff $(p, t)^{(d)}$ the homogeneous component of degree $d$ of Coeff $(p, t)$.

Let $t \in \mathrm{M}_{d}\left(\mathbb{F}_{2}\left[y_{1}, \ldots, y_{n / 2}\right]\right)$, and we can deduce from $(5)$ that $\operatorname{Coeff}(\tilde{p}, t)^{(d)}=$ $0=\operatorname{Coeff}(\tilde{q}, t)^{(d)}$. Then, the homogeneous component of degree $d$ of $\operatorname{Coeff}(p, t)$ (resp. Coeff $(q, t))$ is equal to the sum of the contributions with terms of degree $d$ that each monomial $m_{i}$ (resp. $m_{j}^{\perp}$ ) present in (8) makes to, respectively, $\operatorname{Coeff}(p, t)^{(d)}$ and $\operatorname{Coeff}(q, t)^{(d)}$, this is,

$$
\begin{aligned}
& \operatorname{Coeff}(p, t)^{(d)}=\alpha_{1} \operatorname{Coeff}\left(m_{1}, t\right)^{(d)}+\ldots+\alpha_{N_{d}} \operatorname{Coeff}\left(m_{N_{d}}, t\right)^{(d)}, \\
& \operatorname{Coeff}(q, t)^{(d)}=\beta_{1} \operatorname{Coeff}\left(m^{\perp}{ }_{1}, t\right)^{(d)}+\ldots+\beta_{N_{d}} \operatorname{Coeff}\left(m^{\perp}{ }_{N_{d}}, t\right)^{(d)} .
\end{aligned}
$$

The fact that $G$ and $G^{T}$ have the same entries (in different positions) and are involved in the evaluations of $p_{i}\left(\left(y_{1}, \ldots, y_{n / 2}\right)(I \mid G)\right)$ and $q_{j}\left(\left(y_{1}, \ldots, y_{n / 2}\right)\left(G^{T} \mid I\right)\right)$ 
produces certain relations between expressions of the form $\operatorname{Coeff}\left(m_{i}, t_{1}\right)^{(d)}$ and expressions of the form $\operatorname{Coeff}\left(m^{\perp}{ }_{j}, t_{2}\right)^{(d)}$ for appropriate $t_{1}, t_{2} \in \mathrm{M}_{d}\left(\mathbb{F}_{2}\left[y_{1}, \ldots\right.\right.$, $\left.\left.y_{n / 2}\right]\right)$. These relations are detailed in the following result:

Proposition 7. Let $(p, q) \in \mathbb{F}_{2}[\boldsymbol{x}] \times \mathbb{F}_{2}[\boldsymbol{x}]$ be polynomials of degreed such that $p$ vanishes on a vector subspace $A \subset \mathbb{F}_{2}^{n}$ of dimension $n / 2$ and $q$ vanishes on the orthogonal space $A^{\perp}$. Let $N_{d}=\left(\begin{array}{c}n / 2 \\ d\end{array}\right)$. For all $i, j \in\left\{1, \ldots, N_{d}\right\}$, it holds that:

$$
\operatorname{Coeff}\left(m_{i}, t_{j}\right)^{(d)}=\operatorname{Coeff}\left(m^{\perp}{ }_{j}, t_{i}\right)^{(d)},
$$

where $t_{1}<\ldots<t_{N_{d}}, m_{1}<\ldots<m_{N_{d}}$, and $m^{\perp}{ }_{1}<$ $\ldots<m^{\perp} N_{d}$ are ordered increasingly in the sets of monomials $\mathrm{M}_{d}\left(\mathbb{F}_{2}\left[y_{1}, \ldots, y_{n / 2}\right]\right), \mathrm{M}_{d}\left(\mathbb{F}_{2}\left[x_{n / 2+1}, \ldots, x_{n}\right]\right), \quad$ and $\quad \mathrm{M}_{d}\left(\mathbb{F}_{2}\left[x_{1}, \ldots, x_{n / 2}\right]\right)$ respectively. Also, $\operatorname{Coeff}\left(m_{i}, t_{j}\right)$ (resp. $\left.\operatorname{Coeff}\left(m^{\perp}{ }_{j}, t_{i}\right)\right)$ denotes the coefficient of $t_{j} \in \mathrm{M}_{d}\left(\mathbb{F}_{2}\left[y_{1}, \ldots, y_{n / 2}\right]\right)$ (resp. $t_{i} \in \mathrm{M}_{d}\left(\mathbb{F}_{2}\left[y_{1}, \ldots, y_{n / 2}\right]\right)$ ) in $m_{i}\left(\left(y_{1}, \ldots, y_{n / 2}\right)(I \mid G)\right)$ (resp. $\left.m^{\perp}{ }_{j}\left(\left(y_{1}, \ldots, y_{n / 2}\right)\left(G^{T} \mid I\right)\right)\right)$. Finally, the expression $\operatorname{Coeff}\left(m_{i}, t_{j}\right)^{(d)}$ (resp. Coeff $\left.\left(m^{\perp}{ }_{j}, t_{i}\right)^{(d)}\right)$ denotes the homogeneous component of degree d of Coeff $\left(m_{i}, t_{j}\right)$ (resp. Coeff $\left.\left(m^{\perp}{ }_{j}, t_{i}\right)\right)$.

Proof. Given $i, j \in\left\{1, \ldots, N_{d}\right\}$, we have that $t_{i}=y_{i_{1}} y_{i_{2}} \ldots y_{i_{d}}, t_{j}=y_{j_{1}} y_{j_{2}} \ldots y_{j_{d}}$, for some $i_{1}, i_{2}, \ldots, i_{d}$, $j_{1}, j_{2}, \ldots, j_{d} \in\{1, \ldots, n / 2\}$. On the one hand:

$$
\operatorname{Coeff}\left(m_{i}, t_{j}\right)^{(d)}=\operatorname{Coeff}\left(\prod_{k=1}^{d} x_{i_{k}+n / 2}, y_{j_{1}} y_{j_{2}} \ldots y_{j_{d}}\right)^{(d)}
$$

Observing the system (5), this is the coefficient of $y_{j_{1}} y_{j_{2}} \ldots y_{j_{d}}$ in the product

$$
\prod_{k=1}^{d} \sum_{\ell=0}^{n / 2-1} g_{\ell+1, i_{k}} y_{\ell+1}
$$

which, after expanding it, equals

$$
\sum_{\pi \text { permutation over }\{1, \ldots, d\}} \prod_{k=1}^{d} g_{j_{\pi(k)}, i_{k}} .
$$

On the other hand,

$$
\operatorname{Coeff}\left(m^{\perp}{ }_{j}, t_{i}\right)^{(d)}=\operatorname{Coeff}\left(\prod_{k=1}^{d} x_{j_{k}}, y_{j_{1}} y_{j_{2}} \ldots y_{j_{d}}\right)^{(d)}
$$

Again, observing the system (5), this is the coefficient of $y_{i_{1}} y_{i_{2}} \ldots y_{i_{d}}$ in the product

$$
\prod_{k=1}^{d} \sum_{\ell=1}^{n / 2} g_{j_{k}, \ell} y_{\ell}
$$


which, again after expanding it, equals

$$
\sum_{\pi \text { permutation over }\{1, \ldots, d\}} \prod_{k=1}^{d} g_{j_{k}, i_{\pi(k)}} .
$$

Now (10) and (9) clearly coincide since

$$
\begin{aligned}
& \sum_{\pi \text { permutation over }\{1, \ldots, d\}} \prod_{k=1}^{d} g_{j_{k}, i_{\pi(k)}}=\sum_{\pi \text { permutation over }\{1, \ldots, d\}} \prod_{k=1}^{d} g_{j_{\pi}-1(\pi(k))}, i_{\pi(k)}= \\
& \sum_{\pi^{-1} \text { permutation over }\{1, \ldots, d\}} \prod_{k=1}^{d} g_{j^{-1}(k)}, i_{k} .
\end{aligned}
$$

We can use this proposition to identify the suitable linear combinations that are of degree $d-1$ :

Theorem 5. Let the notations be as in Proposition 7. There exist $i, j \in\left\{1, \ldots, N_{d}\right\}$ such that the equation

$$
\operatorname{Coeff}\left(p, t_{j}\right)+\operatorname{Coeff}\left(q, t_{i}\right)+\sum_{\left\{k \neq i \mid \alpha_{k} \neq 0\right\}} \operatorname{Coeff}\left(q, t_{k}\right)+\sum_{\left\{\ell \neq j \mid \beta_{\ell} \neq 0\right\}} \operatorname{Coeff}\left(p, t_{\ell}\right)
$$

is of degree $d-1$.

Proof. Denote by $i, j$ the smallest indexes such that $\alpha_{i}, \beta_{j} \neq 0$. Using Proposition 7,

$$
\operatorname{Coeff}\left(m_{i}, t_{j}\right)^{(d)}=\operatorname{Coeff}\left(m^{\perp}{ }_{j}, t_{i}\right)^{(d)} .
$$

Now, for every $k \neq i$ such that $\alpha_{k} \neq 0$ and for all $\ell \neq j$ such that $\beta_{\ell} \neq 0$, using Proposition 7 we get the following equalities:

$$
\begin{gathered}
\operatorname{Coeff}\left(m_{k}, t_{j}\right)^{(d)}=\operatorname{Coeff}\left(m^{\perp}{ }_{j}, t_{k}\right)^{(d)}, \operatorname{Coeff}\left(m^{\perp}{ }_{\ell}, t_{i}\right)^{(d)}=\operatorname{Coeff}\left(m_{i}, t_{\ell}\right)^{(d)}, \text { and } \\
\operatorname{Coeff}\left(m^{\perp}{ }_{\ell}, t_{k}\right)^{(d)}=\operatorname{Coeff}\left(m_{k}, t_{\ell}\right)^{(d)} .
\end{gathered}
$$

Now adding up both the left-hand side and the right-hand side of all the four equalities, we obtain

$$
\begin{aligned}
& \operatorname{Coeff}\left(m_{i}, t_{j}\right)^{(d)}+\sum_{\left\{k \neq i \mid \alpha_{k} \neq 0\right\}} \operatorname{Coeff}\left(m_{k}, t_{j}\right)^{(d)}+\sum_{\left\{\ell \neq j \mid \beta_{\ell} \neq 0\right\}} \operatorname{Coeff}\left(m^{\perp}, t_{i}\right)^{(d)}+ \\
& +\sum_{\left\{\ell \neq j, k \neq i \mid \alpha_{k} \neq 0, \beta_{\ell} \neq 0\right\}} \operatorname{Coeff}\left(m^{\perp}{ }_{\ell}, t_{k}\right)^{(d)}+\operatorname{Coeff}\left(m^{\perp}{ }_{j}, t_{i}\right)^{(d)}+\sum_{\left\{k \neq i \mid \alpha_{k} \neq 0\right\}} \operatorname{Coeff}\left(m^{\perp}{ }_{j}, t_{k}\right)^{(d)}+ \\
& +\sum_{\left\{\ell \neq j \mid \beta_{l} \neq 0\right\}} \operatorname{Coeff}\left(m_{i}, t_{\ell}\right)^{(d)}+\sum_{\left\{\ell \neq j, k \neq i \mid \alpha_{k} \neq 0, \beta_{\ell} \neq 0\right\}} \operatorname{Coeff}\left(m_{k}, t_{\ell}\right)^{(d)}=0 .
\end{aligned}
$$


The left-hand side of this equality is the homogeneous component of degree $d$ of

$$
\operatorname{Coeff}\left(p, t_{j}\right)+\operatorname{Coeff}\left(q, t_{i}\right)+\sum_{\left\{k \neq i \mid \alpha_{k} \neq 0\right\}} \operatorname{Coeff}\left(q, t_{k}\right)+\sum_{\left\{\ell \neq j \mid \beta_{\ell} \neq 0\right\}} \operatorname{Coeff}\left(p, t_{\ell}\right)
$$

which means that we cancelled out the terms of degree $d$ and so the required equation is of degree $d-1$.

This result can be used to generate low-degree equations:

Corollary 1. Let $\left(\mathbf{p}=\left(p_{1}, \ldots, p_{m}\right), \mathbf{q}=\left(q_{1}, \ldots, q_{m}\right)\right) \in \mathbb{F}_{2}[\boldsymbol{x}]^{m} \times \mathbb{F}_{2}[\boldsymbol{x}]^{m}$ be a degree-d instance of $\mathrm{HSP}_{2}$. We can easily generate $\mathcal{O}\left(m^{2}\right)$ equations of degreed -1 . These equations are linear combinations of the degree-d equations of $\mathrm{Sys}_{\mathrm{HSP}_{2}}$.

Proof. We apply simply Theorem 5 to each pair of polynomials $\left(p_{i}, q_{j}\right) \in \mathbb{F}_{2}[\mathbf{x}] \times$ $\mathbb{F}_{2}[\mathbf{x}]$. From the proof of Theorem 5 , it is clear that these equations are linear combinations of the equations from $\mathrm{Sys}_{\mathrm{HSP}_{2}}$.

To conclude this part, we include below experimental results about the number of equations of degree $d-1$ generated as Corollary 1 which are linearly independent. In the table, we denote by \# eqs $_{\text {pr }}$ the number of linearly independent equations obtained in practice and by $\#$ eqs $_{\text {th }}$ the maximum number of linearly independent equations that can be obtained, which is $m^{2}$.

\begin{tabular}{|l|c|c|c|c|}
\cline { 2 - 5 } \multicolumn{1}{c|}{} & \multicolumn{2}{c|}{$d=3$} & \multicolumn{2}{c|}{$d=4$} \\
\cline { 2 - 5 } \multicolumn{1}{c|}{} & eqs $_{\text {pr }}$ & $\#$ eqs $_{\text {th }}$ & $\#$ eqs $_{\text {pr }}$ & $\#$ eqs $_{\text {th }}$ \\
\hline$m=n=10$ & 99 & 100 & 71 & 100 \\
\hline$m=n=12$ & 144 & 144 & 144 & 144 \\
\hline$m=n=14$ & 196 & 196 & 196 & 196 \\
\hline$m=n=16$ & 256 & 256 & 256 & 256 \\
\hline
\end{tabular}

We observe that the behaviour is unstable for small values of the parameters. This is partially due to the fact that if a polynomial $p_{i}$ (resp. $q_{j}$ ) does not have terms of degree $d$ in $\mathbb{F}_{2}\left[x_{n / 2+1}, \ldots, x_{n}\right]$ (resp. $\mathbb{F}_{2}\left[x_{1}, \ldots, x_{n / 2}\right]$ ), then we do not get equations of degree $d-1$ applying Theorem 5 to the pair $\left(p_{i}, q_{k}\right)$ for all $k \in\{1, \ldots, m\}$ (resp. from the pair $\left(p_{k}, q_{j}\right)$ for all $\left.k \in\{1, \ldots, m\}\right)$. This happens with probability $1 / 2^{\left(\begin{array}{c}n / 2 \\ d\end{array}\right)}$, which is not too small for low parameters. So, for small parameters it is possible that we obtain a number of equations of degree $d-1$ lower than $m^{2}$. However, if this is the case there still are equations of degree $d-1$ (or lower) produced by the terms of degree $d-1$ (or lower). We see that the behavior becomes stable for big enough values of the parameters $m, n$ obtaining as many equations of degree $d-1$ as possible, this is, $m^{2}$. 


\section{Conclusions}

In this paper we presented a very efficient attack for $\mathrm{HSP}_{q}$ with $q>d$. The case $q \leq d$ is not treated, but as there are reductions modulo the field equations taking place, the behaviour might be similar to the one over $\mathbb{F}_{2}$ (Section 4). Since the asymptotic probability of success of this algorithm is $1-1 / q$, the quantum money scheme extended to $\mathbb{F}_{q}$ is not secure for big $q$. We also provided some experimental and theoretical arguments that support the conjecture that $\mathrm{HSP}_{2}$ can be solved in polynomial time. In both cases, we only considered public polynomials chosen randomly as described in [1]. For other choices of public polynomials with a certain structure (for instance, homogeneous polynomials of degree $d, \ldots$ ) the hardness of HSP remains open. Another interesting open question is the hardness of the noisy version of the scheme, which is related to the study of the noise-free version when $m=\mathcal{O}(1)$ (in this paper, we considered $m=\mathcal{O}(n))$.

Acknowledgments. We thank Scott Aaronson and the anonymous referees for detailed comments which greatly improved this work. Jean-Charles Faugère and Ludovic Perret have been partially supported supported the HPAC grant (ANR-11-BS02-013) of the French National Research Agency.

\section{References}

1. Aaronson, S., Christiano, P.: Quantum money from hidden subspaces. In: Proceedings of the 44th Symposium on Theory of Computing Conference, STOC 2012, New York, NY, USA, May 19-22, pp. 41-60 (2012)

2. Bardet, M., Faugère, J.-C., Salvy, B.: On the Complexity of the F5 Gröbner basis Algorithm. Journal of Symbolic Computation, 1-24

3. Bardet, M., Faugère, J.-C., Salvy, B.: On the complexity of Gröbner basis computation of semi-regular overdetermined algebraic equations. In: Proc. of International Conference on Polynomial System Solving (ICPSS), pp. 71-75 (2004)

4. Bardet, M., Faugère, J.-C., Salvy, B., Yang, B.-Y.: Asymptotic behaviour of the degree of regularity of semi-regular polynomial systems. In: Proc. of MEGA 2005, Eighth International Symposium on Effective Methods in Algebraic Geometry (2005)

5. Bennett, C.H., Brassard, G., Breidbard, S., Wiesner, S.: Quantum cryptography, or unforgeable subway tokens. In: Proceedings of CRYPTO, pp. 267-275 (1982)

6. Bosma, W., Cannon, J.J., Playoust, C.: The Magma algebra system I: The user language. Journal of Symbolic Computation 24(3-4), 235-265 (1997)

7. Brent, R.P., McKay, B.D.: Determinants and rank of random matrices over $\mathbb{Z}_{m}$. Discrete Math. 66, 35-50 (1987)

8. Buchberger, B.: Ein Algorithmus zum Auffinden der Basiselemente des Restklassenringes nach einem nulldimensionalen Polynomideal. PhD thesis, University of Innsbruck (1965)

9. Buchberger, B.: Bruno Buchberger's PhD thesis 1965: An algorithm for finding the basis elements of the residue class ring of a zero dimensional polynomial ideal. J. Symb. Comput. 41(3-4), 475-511 (2006)

10. Buchberger, B.: Comments on the translation of my phd thesis. J. Symb. Comput. 41(3-4), 471-474 (2006) 
11. Courtois, N., Meier, W.: Algebraic attacks on stream ciphers with linear feedback. In: Biham, E. (ed.) EUROCRYPT 2003. LNCS, vol. 2656, pp. 345-359. Springer, Heidelberg (2003)

12. Farhi, E., Gosset, D., Hassidim, A., Lutomirski, A., Shor, P.W.: Quantum money from knots, pp. 276-289 (2012)

13. Faugère, J.-C.: A new efficient algorithm for computing Gröbner bases $\left(F_{4}\right)$. Journal of Pure and Applied Algebra 139, 61-88 (1999)

14. Faugère, J.-C.: A new efficient algorithm for computing Gröbner bases without reduction to zero $\left(F_{5}\right)$. In: ACM Press (ed.) International Symposium on Symbolic and Algebraic Computation, ISAAC 2002, pp. 75-83 (2002)

15. Faugère, J.-C., Joux, A.: Algebraic cryptanalysis of hidden field equation (HFE) cryptosystems using Gröbner bases. In: Boneh, D. (ed.) CRYPTO 2003. LNCS, vol. 2729, pp. 44-60. Springer, Heidelberg (2003)

16. Faugère, J.-C., Perret, L.: Polynomial equivalence problems: algorithmic and theoretical aspects. In: Vaudenay, S. (ed.) EUROCRYPT 2006. LNCS, vol. 4004, pp. 30-47. Springer, Heidelberg (2006)

17. Gavinsky, D.: Quantum money with classical verification, pp. 42-52 (2012)

18. Mosca, M., Stebila, D.: Quantum coins. Error-Correcting Codes, Finite Geometry and Cryptography 523, 35-47 (2010)

19. Patarin, J.: Hidden fields equations (HFE) and isomorphisms of polynomials (IP): two new families of asymmetric algorithms. In: Maurer, U.M. (ed.) EUROCRYPT 1996. LNCS, vol. 1070, pp. 33-48. Springer, Heidelberg (1996)

20. Wiesner, S.: Conjugate coding. ACM SIGACT News 15(1), 78-88 (1983) 